\title{
SMART HELMET TO DRIVER ENVIRONMENT INTERACTION SYSTEM FOR MOTORCYCLES
}

\author{
Ms.Therese Sijiya.S \\ M.E.Communication System \\ Rohini College Of Engineering And Technology \\ Kanyakumari, Tamilnadu, India
}

\begin{abstract}
Motorcycles and bikes form an integral part of personalized transportation in India. With the growing number of 2-wheel motor vehicles, frequency of accidents is on the rise, or because he was riding while drunk. A major portion of the fatalities occur because the person was either not wearing a helmet. This paper proposes mechanisms that can detect if one is wearing the helmet, detect accidents, and detect whether the person has over-consumed alcohol. For this purpose, we use onboard sensors - flex sensor, impact sensor, accelerometer (ADXL355) and breath-analyzer (MQ3). The accelerometer measures the change in tilt, in $X Y$ and $Z$ axes respectively, and sends the data to a server via an online application programming interface (API). An intelligent motorcycle helmet that includes two miniature infrared (IR) transceivers, a miniature image sensor (camera), an embedded computation processing (ECP) module, a battery charging module, a microphone, and earphones. Two large truck/bus vehicle registration plate image recognition modes (day and night) are designed and implemented for the purpose of large truck/bus approach intimation. The experimental results show that the intelligent helmet proposed can successfully recognize an image of the vehicle. The breath analyzer senses the amount of alcohol present in the breath of a person wearing the helmet and reports if it is beyond the legal limit. The server also uses the data gathered from the accelerometer and the pressure sensors, to train a support vector machine (SVM). This can help optimize accident detection in the future when enough data is gathered to provide reliable accuracy.
\end{abstract}

Keywords-Smart Helmet, Breath Analyser, Accident Prevention, Accelerometer.

\section{INTRODUCTION}

India is constantly facing challenges in reducing the vehicle accident death rate of which, motorcycle accidents contributes a major share. According to reports, nearly $76 \%$ of such victims sustain head injuries, which is mostly attributed to the non-wearing of helmet and consuming alcohol by the rider while driving. It is stated in the section 129 and section 185 respectively, of the Indian Motor Vehicle Act that, every

\author{
Mr. P.Benesh Selva Nesan, \\ M.E., Assistant Professor \\ Rohini College Of Engineering And Technology \\ Kanyakumari, Tamilnadu, India
}

motor bike rider should put on the helmet; and the blood alcohol content (BAC) of the rider cannot be beyond 0.08 $\mathrm{mg} / \mathrm{L}$. Often, it is observed that the riders tend to disobey these regulations and it becomes very difficult for the traffic police to monitor every rider on the road.

Nowadays there are many accidents occurred in our daily life. This system is mainly for the security purpose and safety for the bike riders against the accidents. The first step in this system is to check whether the helmet has been wear or not, the bike will not start unless the rider wears the helmet for this we go for FSR sensor which will sense the pressure and force. The camera in the bike will check whether the rider had wear the helmet or not. The second step is to identify whether the rider has consumed the alcohol or not. An alcohol sensor will check the alcohol in rider's breath, in order to prevent the accidents due to drunken and driving which cause a lot of accidents. Alcohol sensor is use as breath analyzer which detect the presence of alcohol in rider breathe if it is exceeds permissible range ignition cannot start.

Jacobs et al., (1982) carried out research in third world countries with a view to establish the nature and extent of traffic-related accident problems and to assess the effectiveness of remedial measures in the long term. Significant observations showed that as vehicle ownership increased the fatality rate decreased: that is countries with lower levels of vehicle ownership were those with higher fatality rates. Also the fatality rate increased over a given period of time for the same level of vehicle ownership. Remedial measures focused on the studies of behavior of road users and road user knowledge in developing countries, traffic law enforcement, vehicle safety and highway engineering.

Othman et al., (2009) Crash risk results from interaction between three elements, sometimes called the three traffic safety pillars: road users, vehicles, and infrastructure. Similarly, epidemiologists use the terms host, agent, and environment (Haddon, 1980). Haddon's definition of environment also includes the social environment. Singlevehicle crashes may involve only one vehicle and one road user, whereas conflicts involve an interaction between several vehicles and road users. Depending on the energy that is exchanged between road users, vehicles and infrastructure, crashes may result in injuries with varying levels of severity. Crashes maybe fatal when forces transferred to victims exceed their biomechanical tolerance. This tolerance depends on age, 


\section{International Journal of Engineering Applied Sciences and Technology, 2020 \\ Vol. 4, Issue 11, ISSN No. 2455-2143, Pages 635-638 \\ Published Online March 2020 in IJEAST (http://www.ijeast.com)}

health status, stature and other characteristics of road users involved in a crash.

Shruthi.P et al., (2013) approached her study regarding the road accidents in south Indian metropolitan cities in different dimensions. She researched the injury profile and mortality pattern of the autopsy cases to draw public attention and to create awareness among the public for accident free transportation. The records from the year 2010 t0 2012 was used for the study. From the study it is revealed that around $55 \%$ of cases are accident cases, out of which $78 \%$ are male cases. Moreover it is found that most of the accidents occur in the peak hours of the morning 6.00 AM to 12.00 PM. Around $30.22 \%$ of cases had fatal injuries involving head injuries, abdominal injuries and limb injuries. From this study it is recommended additional attention is needed at the high accident prone areas and strict legislations to prevent and control drunken driving.

The rest of the paper is organized as follows. Proposed algorithm is explained in section II. Experimental results are presented in section III. Concluding remarks are given in section IV.

\section{Proposed AlgorithM}

The proposed Intelligent helmet have transmitter and receiver sides. The transmitter side consists of pressure sensor, breath analyzer and a bluethooth. The receiver side has accelerometer ADXL355, bluthooth, a wifi module and LCD display.It can detect if one is wearing the helmet, detect accidents, and detect whether the person has over-consumed alcohol.The accelerometer we use is a 3-axis accelerometer that measures tilt of the device with respect to the earth, in 3 axes $\mathrm{X}, \mathrm{Y}$ and $\mathrm{Z}$.Accelerometer detects the sudden change of road condition and slow down the vehicle.

Ramya.D et al., (2018) Nowadays accidents are a major cause of death and disability. Public awareness and safety is one of the most important confrontations in the place of effective welfare for the bikers. Bike accidents in highways and roadways is one of the increasing fatality rates for the previous years. With the help of our project we desire to reduce the feasibility of dying because of bike accidents. In this project, we have advanced the two wheelers system by the sensors that monitors and control the speed. The sensor used in our project to control the speed is the speed sensor. Here ultrasonic sensor is also placed in order to maintain the distance between the vehicles to avoid collision between the vehicles. The riders will be given indication when the control of the bike exceeds the particular limit. The microcontroller used in this vehicle controls the whole sensors and devices connected in the speedometer and in the gear box. The red light brightens if the speed goes above $80 \mathrm{~km} / \mathrm{hr}$. Because of this death, accidents and collisions can be evaded. The aim of our project is to initially check whether the person riding the bike is aware about the speed control, distance between the vehicles in order to avoid the hazards.

G. Pavithra et al., (2019) every year in india a large number of deaths occur due to road accidents. Drivers on two wheeler contribute significantly to 134513 deaths. In a large number of two wheeler accidents, deaths occur because no preventive actions have been taken before hand by the driver or those sitting in the two-wheeler. Intention of our project is to prevent the bike riders without wearing helmet and control the maximum two persons only sit on the seat by using embedded system technology. And also we are using breathe analyser, accelerometer and radio frequency system. Those are place in the particular area of field. Now we are adopting this setup using pic16f877a microcontroller in embedded system. So we need the program to control the function of full bike system. By this way of process we must prevent from more number of accidents.

Navjot Kumar et al., (2017) The paper printed in this article highlights the dependence of India on the coal mines outcome to satisfy various needs relating from power plants to domestic fuel applications. The paper also shows a large number of people working for mines throughout the country. The paper focuses on the security concerns and shows the numbers of accidents covering both fatal and non-fatal accidents and forms a base to enhance the security in the underground mines. The research work is carried around using three subsystems mainly miner kit, surface computer and base station.

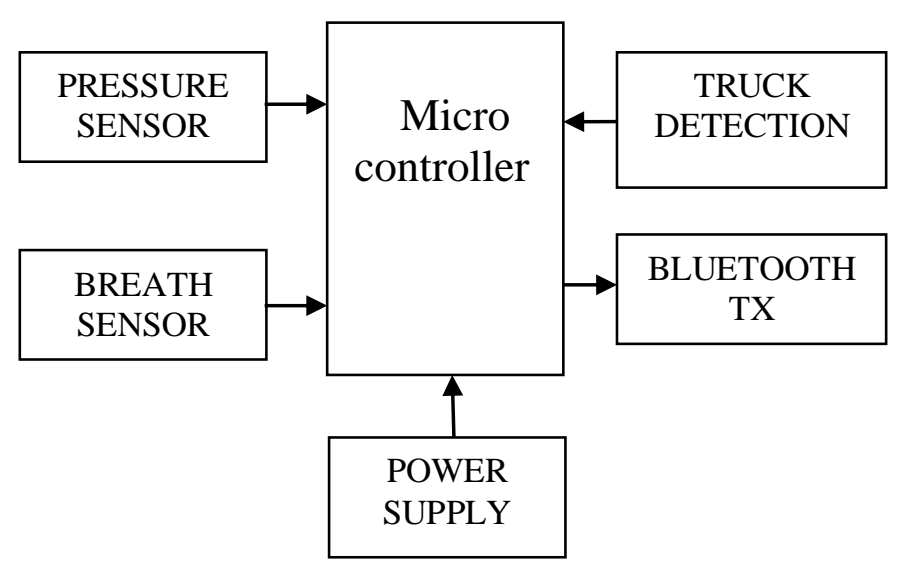

Fig. 1.Block Diagram Of Transmitter 


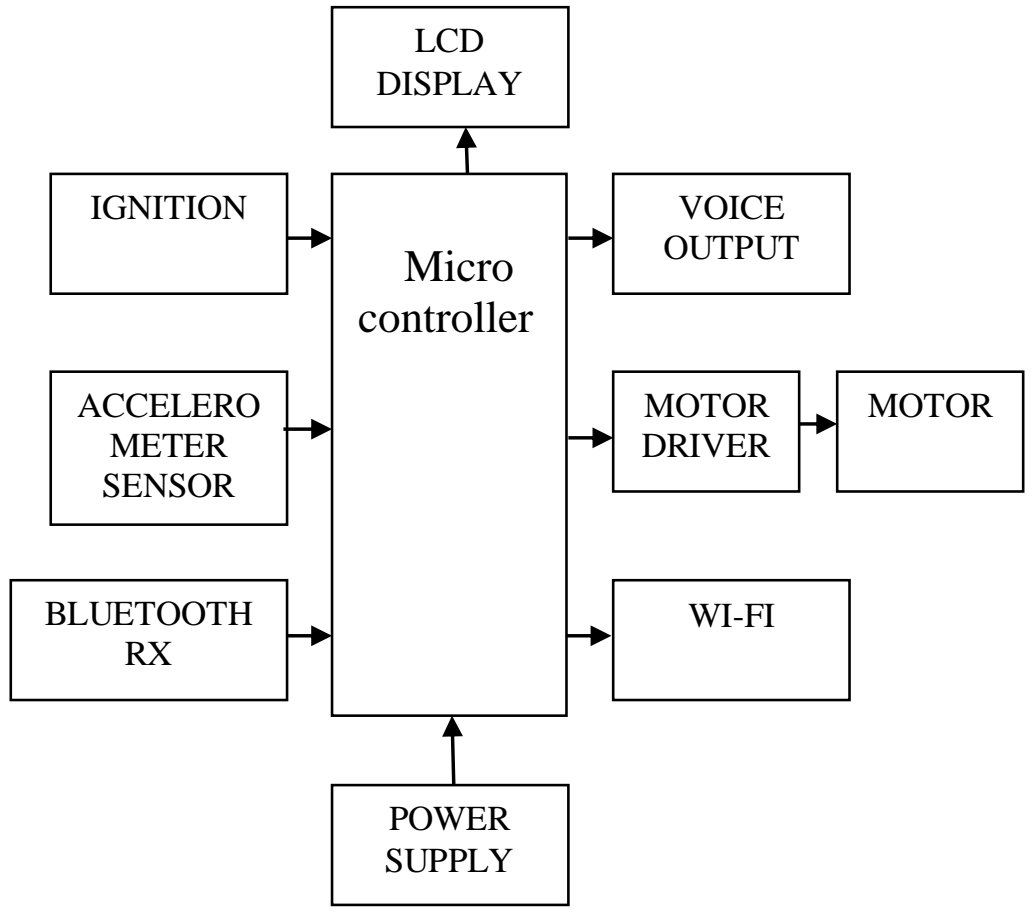

Fig. 2.Block Diagram Of Receiver

The Arduino Uno R3 is a microcontroller board based on the ATmega328. It has 14 digital input/output pins (of which 6 can be used as PWM outputs), 6 analog inputs, a $16 \mathrm{MHz}$ crystal oscillator, a USB connection, a power jack, an ICSP header, and a reset button.Alcohol Gas Sensor is a low-cost semiconductor sensor which, used to detect the presence of alcohol vapour gas at concentrations from $0.05 \mathrm{mg} / \mathrm{L}$ to 10 $\mathrm{mg} / \mathrm{L}$. The L293D is quadruple high-current half-H drivers.It is designed to provide bidirectional drive currents of up to 600$\mathrm{mA}$ at voltages from $4.5 \mathrm{~V}$ to $36 \mathrm{~V} . \mathrm{LCD}$ (liquid crystal display) is the technology used for displays in notebook and other smaller computers. Like light-emitting diode (LED) and gas-plasma technologies, LCDs allow displays to be much thinner than cathode ray tube (CRT) technology.An accelerometer is a device that measures proper acceleration.The ESP8266 Wi-Fi Module is a self contained SOC with integrated TCP/IP protocol stack that can give any microcontroller access to your Wi-Fi network. Bluetooth is a technology for wireless communication. It is designed to replace cable connections.It uses serial communication to communicate with devices. Vehicle detection plays an important role for the localization of an image or robust vehicle detection is the first step in video processing.

\section{EXPERIMENT AND RESULT}

The below figure shows the circuit diagram of intelligent helmet system. The arduino micro controller controls the system. The design has three layer helmet, alcohol and Bluetooth. Pressure sensor connects the digital pin of arduino microcontroller it sense the helmet is lock or unlock. If the helmet is unlock the two wheeler not started. Breath sensor is detect the driver is drink alcohol. Bluetooth reader connects the arduino through the software serial. In the above circuit LCD is used to indicate the status of electrical loads and also used to display received data. Here LCD is interfaced to the Arduino microcontroller in 4 bit mode.

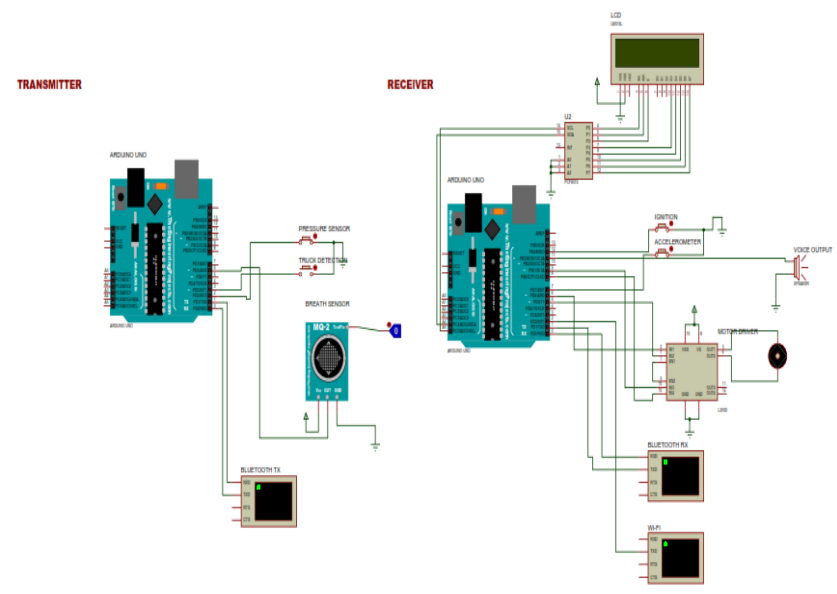

Fig. 3.Circuit Diagram of Proposed System

Wi-Fi module TX and RX pins are connected to the RX and TX pins of controller respectively. Controller communicates with wi-fi module using serial communication (UART protocol). Use a baud rate of 9600 to communicate with wi-fi. The accelerometer uses very little current, so it can be plugged into your board and run directly off of the output from the digital output pins. To do this, you'll use three of the analog input pins as digital $\mathrm{I} / \mathrm{O}$ pins, for power and ground to the accelerometer, and for the self-test pin. You'll use the other three analog inputs to read the accelerometer's analog output. Pressure sensor connects the digital pin of arduino microcontroller it sense the helmet is lock or unlock. LCD is interfaced to the Arduino microcontroller in 4 bit mode. Wi-Fi module TX and RX pins are connected to the RX and TX pins of controller respectively. Controller communicates with wi-fi module using serial communication (UART protocol). analog input pins as digital I/O pins, for power and ground to the accelerometer, and for the self-test pin. If the person is not wearing the helmet then the motorbike will not start. The 3 axis accelerometer detect the pothole in the road and reduce the speed so that one can safely drive over that. 


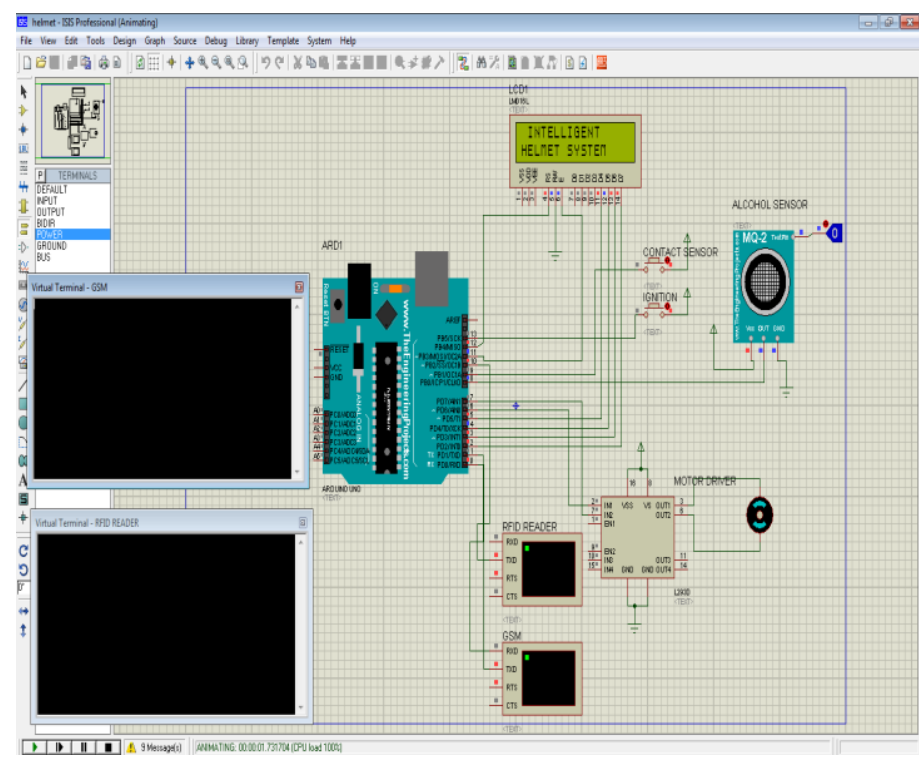

Fig. 4.Stimulation Output Of Proposed System

\section{CONCLUSION}

This helmet system implemented marks a pronounced reduction of power by $\sim 93 \%$ as compared to reported systems using microcontroller. The low power consumption in our system is attributed to several salient properties of the helmet system which primarily includes the incorporation of the timer circuit. The helmet system implemented is fast, compact, feasible and comparatively of lower cost. The helmet system addresses the major causes of two wheeler accident and provides an effective as well as a low power, low cost preventive measure to reduce the devastating effects of road accidents by two-wheelers. The system can play a significant role in improving the safety aspects of the two-wheeler riders.

\section{REFERENCE}

[1] Ajay A., Vishnu G., Kishoreswaminathan V., Vishwanth V., and Scholar U. G. (2017) "Accidental identification and navigation system in helmet," in Proceedings of the 2017 International Conference on Nextgen Electronic Technologies: Silicon to Software (ICNETS2).

[2] Spelta C., Manzoni V., Corti A., Goggi A., and Savaresi S.M. (2010). "Smart phone- based vehicle- to driver/environment interaction system for motorcycles," IEEE Embedded Systems Letters, vol. 2, no. 2, (pp. 3942).

[3] Yang C.-Y., Wu C.-F., Samani H., and Lien P.W.( 2013)."Ergonomic design of an active alert helmet," in Proceeding of the 2013 1st International Conference on Orange Technologies (ICOT).

[4] Sasikala G., Padol K., Katekar A. A., and Dhanasekaran S.( 2015). "Safeguarding of motorcyclists through helmet recognition," in Proceedings of the 2015 International Conference on Smart Technologies and Management for Computing, Communication, Controls, Energy and Materials (ICSTM'15),( pp. 609-612).

[5] Jo J., and Kim H.( 2017). "Development of a safety index to identify differences in safety performance by postal delivery motorcyclists based either in different regional post offices or within the same regional office," International Journal of Geo-Information, vol. 6, article 324, (pp. 1-20).

[6] Li L., Yang J., Otte D., Zhou X., Zhang v., Yu X., and Li W.( 2012). "A study on motor-scooter accidents in China and Germany," in Proceedings of the 2013 Fifth Conference on Measuring Technology and Mechatronics automation, (pp. 110-113).

[7] Rasli M. K. A. M., Madzhi N. K., and Johari J.( 2013). "Smart helmet with sensors for accident prevention," in Proceedings of the 2013 International Conference on Electrical, Electronics and System Engineering, (pp. 2126).

[8] Rupanagudi S. R., Bharadwaj S., Bhat V. G., Eshwari S., Shreyas S., Aparna B. S., Venkatesan A., Shadnilya A., Subrahmanya V., and Jabeen F.( 2015). "A novel video processing based smart helmet for rear vehicle intimation \& collision avoidance," in Proceedings of the 2015 International Conference on Computing and Network Communications (CoCoNet'15), ( pp. 799-805).

[9] Patil S., Hegde M. G., Bhattacharjee S., and Rajeshwari B. C.( 2016). "Smart motorcycle security system," in Proceedings of the International Conference on Emerging Trends in Engineering, Technology, and Science (ICETETS'16), ( pp. 1-4).

[10] Tapadar S., Ray S., Saha H. N., Saha A. K., and Karlose R.( 2018). "Accident and alcohol detection in Bluetooth enabled smart helmets for motorbikes," in Proceedings of the 2018 IEEE 8th Annual Computing and Communication Workshop and Conference (CCWC'18).

[11] Hsieh T.M., Tsai T.C., Liu Y.W., and Hsieh C.H.( 2016). "How does the severity of injury vary between motorcycle and automobile accident victims who sustain high-grade blunt hepatic and/or splenic injuries? results of a retrospective analysis," International Journal of Environmental Research and Public Health, vol. 17, article 739, (pp. 1-9).

[12] North T., Wagner M., Bourquin S., and Kilcher L.( 2016). "Compact and high-brightness helmet-mounted head-up display system by retinal laser projection," IEEE Journal of Display Technology, vol. 12, no. 9, (pp. 982-985).

[13] Manzoni V., Corti A., Spelta C., and Savaresi S. M.( 2010). "A driver-to-infrastructure interaction system for motorcycles based on smartphone," in Proceedings of the 2013 13th International IEEE Annual Conference on Intelligent Transportation Systems, (pp. 1442-1447). 